\title{
Feedback analysis of transimpedance operational amplifier circuits
}

\author{
Bruun, Erik
}

Published in:

I E E E Transactions on Circuits and Systems Part 1: Regular Papers

Link to article, DOI:

$10.1109 / 81.224302$

Publication date:

1993

Document Version

Publisher's PDF, also known as Version of record

Link back to DTU Orbit

Citation (APA):

Bruun, E. (1993). Feedback analysis of transimpedance operational amplifier circuits. I E E E Transactions on Circuits and Systems Part 1: Regular Papers, 40(4), 275-278. https://doi.org/10.1109/81.224302

\section{General rights}

Copyright and moral rights for the publications made accessible in the public portal are retained by the authors and/or other copyright owners and it is a condition of accessing publications that users recognise and abide by the legal requirements associated with these rights.

- Users may download and print one copy of any publication from the public portal for the purpose of private study or research.

- You may not further distribute the material or use it for any profit-making activity or commercial gain

- You may freely distribute the URL identifying the publication in the public portal

If you believe that this document breaches copyright please contact us providing details, and we will remove access to the work immediately and investigate your claim. 


\section{REFERENCES}

[1] R. Schaumann, K. R. Laker, and M. S. Ghausi, Design of Analog Filters: Passive, Active RC and Switched-Capacitor. Englewood Cliffs, NJ: Prentice-Hall, 1990.

[2] K. Martin and A. S. Sedra, "Effects of the op-amp finite gain and bandwidth on the performance of switched-capacitor filters," IEEE Trans. Circuits Syst., vol. CAS-28, pp. 822-829, August 1981.

[3] C. S. Park and R. Schaumann, "A high-frequency CMOS linear transconductance element," IEEE Trans. Circuits Syst., vol. CAS-33, pp. 1132-1138, 1986.

[4] R. Schaumann and M. A. Tan, "Continuous-time filter," in C. Toumazou, F. J. Lidgey, and D. G. Haigh, eds., Analogue IC Design: The CurrentMode Approach, ch. 9, pp. 371-386. London: Peter Perenigus, 1990.

[5] E. Sánchez-Sinencio, R. L. Geiger, and H. Nevarez-Lozano, "Generation of continuous-time two integrator loop OTA filter structures," IEEE Trans. Circuits Syst., vol. 35, pp. 936-946, Aug. 1988

[6] J. B. Hughes, "Switched-current filters," in C. Toumazou, F. J. Lidgey, and D. G. Haigh, eds., Analogue IC Design: The Current-Mode Approach, ch. 11, pp. 415-450. London: IEE, 1990.

[7] H. Khorramabadi and P. R. Gray, "High-frequency CMOS continuoustime filters," IEEE J. Solid-State Circuits, vol. SC-19, pp. 939-948, Dec 1984.

[8] C. S. Park and R. Schaumann, "Design of a $4 \mathrm{MHz}$ analog integrated CMOS Transconductance-C bandpass filter," IEEE J. Solid-State Circuits, vol. SC-23, pp. 987-996, Aug. 1988

\section{Feedback Analysis of Transimpedance Operational Amplifier Circuits}

Erik Bruun

Abstract-The transimpedance or current feedback operational amplifier (CFB op-amp) is reviewed and compared to a conventional voltage mode op-amp using an analysis emphasizing the basic feedback characteristics of the circuit. With this approach the paradox of the constant bandwidth obtained from CFB op-amps is explained. It is demonstrated in a simple manner that the constant gain-bandwidth product of the conventional op-amp and the constant bandwidth of the CFB op-amp are both in accordance with basic feedback theory and that the differences between the traditional op-amp and the CFB op-amp are due to different ways of controlling the closed-loop gain. For the traditional op-amp the closed-loop gain is altered by altering the loop gain whereas the closedloop gain in a CFB op-amp configuration is altered by altering the input attenuation to the feedback loop while maintaining a constant-loop gain.

\section{INTRODUCTION}

The transimpedance or current feedback operational amplifier (CFB op-amp) as introduced by Nelson and Evans [1] has been available as a monolithic op-amp for a number of years. One of the most prominent features of this amplifier is the constant bandwidth, independent of the closed-loop voltage gain in a feedback configuration. This characteristic has been treated in the literature as a property almost violating traditional feedback theory that prescribes

Manuscript received September 3, 1992; revised January 6, 1993. This paper was recommended by Associate Editor B. S. Song.

E. Bruun is with the Electronics Institute, Building 349, Technical University of Denmark, DK-2800 Lyngby, Denmark.

IEEE Log Number 9208194 that an amplifier with feedback has a constant product of closedloop gain and bandwidth [2], [3]. Hence, an analysis of the CFB amplifier with reference to familiar concepts in feedback theory seems appropriate.

\section{The Voltage Mode Operational Amplifier}

Fig. 1 shows a traditional voltage mode op-amp in both an inverting and a noninverting feedback configuration. Assuming that the opamp differential voltage gain is $A_{d}(s)$ we find the signal flow graphs shown in Fig. 2. From these we find the closed loop $A(s)$ and the loop gain $T(s)$ :

$$
\begin{aligned}
& A(s)=\frac{\alpha A_{d}(s)}{1+T(s)} \\
& T(s)=-\beta A_{d}(s) .
\end{aligned}
$$

For the inverting amplifier we note that

$$
\begin{aligned}
& \alpha=\frac{-R_{2}}{R_{1}+R_{2}} \\
& \beta=\frac{-R_{1}}{R_{1}+R_{2}} .
\end{aligned}
$$

For the noninverting amplifier we find

$$
\begin{aligned}
& \alpha=1 \\
& \beta=\frac{-R_{1}}{R_{1}+R_{2}} .
\end{aligned}
$$

Assuming that $A_{d}(s)=A_{0} /\left(1+s / w_{c}\right)$ we find the closed-loop gain and bandwidth relations

$$
2 \pi B W=\omega_{c}\left(1-3 A_{0}\right)
$$

and

$$
2 \pi G B W=\alpha \omega_{c} A_{0} .
$$

For the noninverting amplifier of Fig. 1(b) with $\alpha=1$ the latter expression shows that the product of gain $G$ and bandwidth $B W$ is constant and equal to the unity gain bandwidth $A_{0} \omega_{c} / 2 \pi$ for the op-amp. For the inverting amplifier (8) shows that the product of gain and bandwidth is actually not constant as $\alpha$ is dependent on the closed-loop gain. Assuming $A_{0} \gg 1$ we note that the low frequency gain is $G=-\alpha / \beta=-R_{2} / R_{1}$ implying that (8) for the inverting configuration results in

$$
2 \pi G B W=\frac{1}{1+1 /|G|} \omega_{c} A_{0} .
$$

Only for $|G| \gg 1$ does this equation express a constant gainbandwidth product.

An important property of feedback is that it reduces distortion, sensitivity to component variations, etc., with a factor of $F(s)=$ $1+T(s)$. For the configurations based on voltage mode operational amplifiers we find

$$
\begin{aligned}
& F(s) \\
& =1+\frac{R_{1}}{R_{1}+R_{2}} \times \frac{A_{0}}{1+s / \omega_{c}} \\
& \simeq \begin{cases}\frac{A_{0} /(|G|+1)+s / \omega_{c}}{1+s / \omega_{c}} & \text { inverting amplifier } \\
\frac{A_{0} / G+s / \omega_{c}}{1+s / \omega_{c}} & \text { noninverting amplificr. }\end{cases}
\end{aligned}
$$

Obviously, $F(s)$ is dependent on the gain $G$ and hence the improvements in distortion, sensitivity, etc., are strongly dependent on $G$ (approximately inversely proportional to $G$ ). 


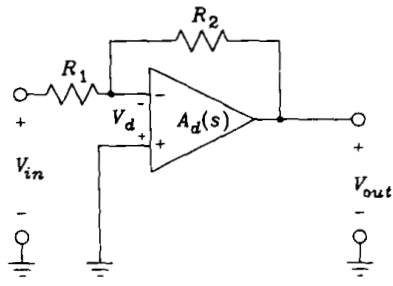

(a)

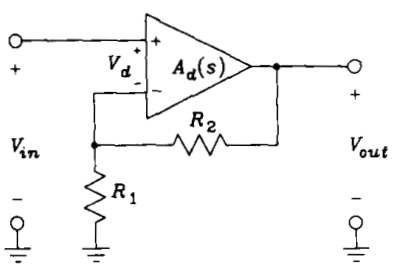

(b)

Fig. 1. (a) Inverting op-amp configuration. (b) Noninverting op-amp configuration.

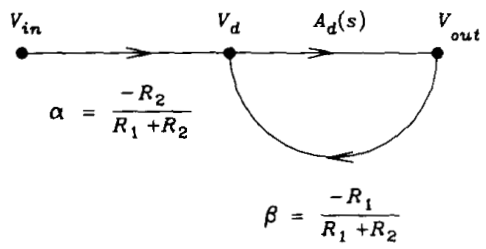

(a)

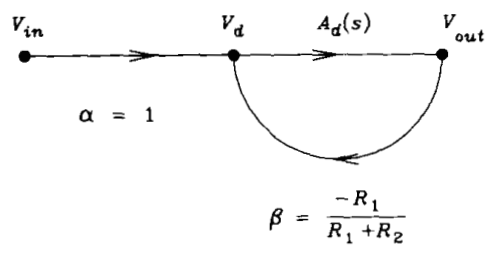

(b)

Fig. 2. Signal flow graphs for (a) inverting voltage mode op-amp configuration and (b) noninverting voltage mode op-amp configuration.

\section{The Current FeedBack Amplifier}

The current feedback operational amplifier is a transimpedance amplifier with the simplified equivalent diagram shown in Fig. 3. In contrast to a conventional voltage mode op-amp the CFB op-amp does not provide a direct differential voltage gain. Rather, it creates a voltage gain by sensing the current flowing into the inverting input and impressing a mirror of the input current onto a high impedance node. It should be noted that the inputs to the CFB op-amp are nonsymmetric. The noninverting input is a high impedance voltage mode input and the inverting input is a low impedance current mode input. The CFB op-amp can be used in exactly the same configurations as shown in Fig. 1. However, the signal flow graphs for the current feedback configurations have very different branch

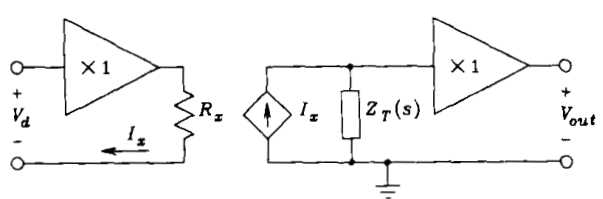

Fig. 3. Simplified block diagram of CFB op-amp.

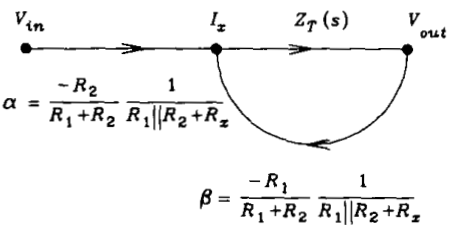

(a)

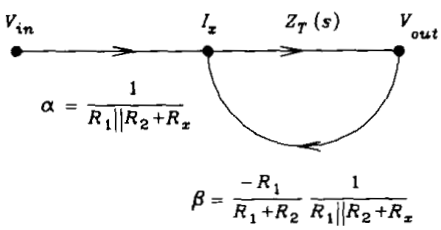

(b)

Fig. 4. Signal flow graphs for (a) inverting CFB op-amp configuration and (b) noninverting CFB op-amp configuration.

transmittances. Fig. 4 shows the signal flow graphs for the CFB configurations. From these graphs we find, using the conventional feedback notation,

$$
A(s)=\alpha \frac{Z_{T}}{1-Z_{T}(s)} \frac{\beta}{1-\beta}
$$

with

$$
\alpha=\left\{\begin{array}{l}
\frac{-R_{2}}{\overline{R_{1}+R_{2}}} \times \frac{1}{R_{1} \| R_{2}+R_{x}}=\frac{-R_{2}}{R_{1} R_{2}+R_{x}\left(R_{1}+R_{2}\right)} \\
\text { inverting amplifier } \\
\frac{1}{R_{1} \| R_{2}+R_{x}}=\frac{R_{1}+R_{2}}{R_{1} R_{2}+R_{x}\left(R_{1}+R_{2}\right)} \\
\text { noninverting amplifier }
\end{array}\right.
$$

and

$$
\beta=\frac{-R_{1}}{R_{1}+R_{2}} \times \frac{1}{R_{1} \| R_{2}+R_{x}}=\frac{-R_{1}}{R_{1} R_{2}+R_{x}\left(R_{1}+R_{2}\right)} .
$$

Assuming $Z_{T}$ to be a parallel connection of a resistor $R_{T}$ and a capacitor $C_{T}$ we have

$$
Z_{T}(s)=\frac{R_{T}}{1+s / \omega_{c}}
$$

with $\omega_{c}=\left(R_{T} C_{T}\right)^{-1}$. The closed-loop gain and bandwidth relations for the CFB amplifier are then calculated as

$$
2 \pi B W=\omega_{c}\left(1-\beta R_{T}\right)
$$

and

$$
2 \pi G B W=\alpha w_{c} R_{T}
$$

These expressions are the same as (7) and (8) for the voltage mode op-amp with $A_{0}$ replaced by $R_{T}$. For the voltage mode op-amp a and $\beta$ are dimensionless whereas for the CFB op-amp they have 
the dimension of $\Omega^{-1}$. Inserting (12) and (13) in (15) the following bandwidth relations result:

$$
\begin{aligned}
2 \pi B W= & \omega_{c}\left(1+\frac{R_{1} R_{T}}{R_{1} R_{2}+R_{x}\left(R_{1}+R_{2}\right)}\right) \\
= & \left\{\begin{array}{c}
\omega_{c}\left(1+\frac{R_{T}}{R_{2}+R_{x}(1+|G|)}\right) \\
\text { inverting amplificr } \\
\omega_{c}\left(1+\frac{R_{T}}{R_{2}+R_{x} G}\right) \\
\text { noninverting amplificr. }
\end{array}\right.
\end{aligned}
$$

Assuming

$$
R_{T} \gg R_{2}+R_{x}|G|
$$

and

$$
R_{x} \ll R_{2} / G
$$

this reduces to the constant bandwidth equation

$$
2 \pi B W=\omega_{c}\left(1+\frac{R_{T}}{R_{2}}\right) \simeq \frac{1}{C_{T} R_{2}} .
$$

Equation (20) shows that the bandwidth $B W$ is independent of the gain $G$ provided $R_{2}$ is left unchanged when changing $G$. We now notice that the important difference between the voltage mode op-amp and the CFB op-amp is that in the voltage mode op-amp configuration the closed-loop gain is changed by changing $\beta$ and hence the loop gain and bandwidth whereas in the CFB op-amp configuration the closed-loop gain is changed by changing $\alpha$ while keeping $\beta$ and hence the loop gain and bandwidth constant. Intuitively, this might be explained as follows: In a conventional voltage mode op-amp configuration the closed-loop gain is controlled by the attenuation of the output voltage by the feedback network. A large attenuation (small $\beta$ ) leads to a large gain. In the CFB op-amp configuration the closed-loop gain is controlled by the attenuation of the input signal such that a large attenuation (small $\alpha$ ) results in a small closed-loop gain. It might seem strange that the noninverting input configuration of Fig. 1(b) has an input attenuation. However, this is explained by the fact that there is an implicit conversion of the input voltage to a current and that the conversion is given by $\left(R_{1} \| R_{2}+R_{x}\right)^{-1}$, i.e., by $\left(R_{2} / G+R_{x}\right)^{-1}$.

With this explanation in mind a relevant question is whether the CFB op-amp attains its properties at the expense of a reduced loop gain and thus a reduced improvement in distortion, sensitivity, etc. From (13) we find

$$
\begin{aligned}
& F(s)=1+T(s)=1-\beta Z_{T}(s) \\
& =1+\frac{R_{1}}{1+s / \omega_{c}} \times \frac{R_{1}}{R_{1} R_{2}+R_{x}\left(R_{1}+R_{2}\right)} \\
& \simeq\left\{\begin{array}{l}
\frac{\frac{R_{T}}{R_{2}+R_{x}(|G|+1)}+s / \omega_{c}}{1+s / \omega_{c}} \\
\text { inverting amplifier } \\
\frac{R_{T}}{R_{2}+R_{x} G+s / \omega_{c}} \\
1+s / \omega_{c} \\
\text { noninverting amplifier. }
\end{array}\right.
\end{aligned}
$$

The bandwidth of $F(s)$ is $\omega_{c}$ just as for the voltage mode op-amp, compare (10). Comparing the noninverting amplifiers we find a low frequency value of $F(s)$ of $A_{0} / G$ for the voltage mode op-amp and $R_{T} /\left(R_{2}+R_{x} G\right)$ for the CFB op-amp. With conventional amplifier architectures employing a single high gain stage with a very high load impedance (e.g., a cascode amplifier with an output buffer) we may assume $A_{0}$ to be on the order of $g_{m} / g_{0}$ where $g_{m}$ is a device transconductance and $g_{o}$ is a device output conductance, a device being a transistor or a compound device (e.g., a cascode circuit). Hence,

$$
F(0)=\frac{A_{0}}{G} \simeq \frac{g_{m}}{g_{0} G}
$$

for the voltage mode op-amp. Similarly, we often find $R_{x} \simeq g_{m}^{-1}$ and $R_{T} \simeq g_{o}^{-1}$ for the CFB op-amp, hence

$$
F(0)=\frac{R_{T}}{R_{2}+R_{x} G} \simeq \frac{g_{m}}{g_{0}\left(G+R_{2} g_{m}\right)} .
$$

Comparing (22) and (23) we note that with equal values of $g_{m}$ and $g_{o}$ for the voltage mode op-amp and the CFB op-amp the voltage mode op-amp indeed has a larger value of $F(s)$. We also note that the difference is insignificant if $R_{2} g_{m}<G$ but this conflicts with the constant bandwidth requirement (19). Thus, the CFB op-amp sacrifices loop gain if the feedback resistor is designed to provide a gain independent bandwidth.

For both types of amplifiers $\omega_{c}$ is on the order of $g_{o} / C_{T}$ where $C_{T}$ is a compensation capacitor. This leads to a bandwidth of

$$
2 \pi B W=\frac{g_{m}}{C_{T} G}
$$

for the voltage mode op-amp and

$$
2 \pi B W=\frac{g_{m}}{C_{T}\left(G+R_{2} g_{m}\right)}
$$

for the CFB op-amp. With equal values of $g_{m}$ and $C_{T}$ we find that the CFB op-amp actually sacrifices bandwidth compared to the voltage mode op-amp.

A similar comparison can be made for the inverting amplifier configurations, leading to the same conclusion.

Often, however, $C_{T}$ is selected smaller for the CFB op-amp than for a comparable voltage mode op-amp. This is due to the fact that a voltage mode op-amp is often compensated to allow its use in a unity gain configuration, i.e., $\omega_{c}$ has been set by proper choice of the dominant pole to a frequency yielding an adequate phase margin when the feedback loop is closed. If the voltage mode op-amp is then used in a feedback configuration with a higher closed-loop gain the bandwidth will decrease in proportion to the gain increase as indicated by $(8)$ provided $\omega_{c}$ is left unchanged. However, with a reduction in the loop gain $T(s)$ the open-loop bandwidth $\omega_{c}$ may be increased correspondingly while maintaining the same phase margin. Hence, the closed-loop bandwidth can be kept at a constant value. However, often the possibility of increasing $\omega_{r}$ by decreasing a capacitor value is not present because the compensation capacitor has been included on-chip with the op-amp. For the CFB op-amp configuration a similar possibility of a decrease in compensation capacitor with an increase in closed-loop gain does not exist if the loop gain $T(s)$ has been designed to be independent of the closed-loop gain. If the bandwidth has been designed to be limited by $R_{x} G$ rather than by $R_{2}$ we find from (17) and (18) that the CFB op-amp in the noninverting configuration has a bandwidth of

$$
2 \pi B \mathrm{H}^{-}=\frac{1}{C_{T} R_{r} G}
$$

which with $R_{x} \simeq g_{m}^{-1}$ is the same as (24), valid for the voltage mode op-amp. In this situation the compensation capacitor may be optimized just as in a voltage mode op-amp.

With these observations in mind one might be inclined to conclude that the CFB op-amp provides little-if any-improvement over a conventional voltage mode op-amp. However, one important feature of the CFB op-amp in most architectures available today [3], [4] is a very high slew rate. This is related to the specific realization of the CFB input stage and cannot be achieved in a conventional voltage mode op-amp amplifier architecture. Thus, the primary advantage of the CFB op-amp is its superior slew rate performance. A secondary advantage of the CFB op-amp is an easier optimization of the frequency response of the loop gain because there is only one highimpedance node in the feedback loop whereas voltage mode op-amps 
may have more than one high impedance node, even in the case of single gain stage op-amps, because of the high impedance of the inverting input.

\section{CONCLUSION}

A comparison of a traditional voltage mode op-amp and a current feedback op-amp has been made for an inverting and a noninverting amplifier configuration. It is concluded that the constant bandwidth feature often associated with the CFB op-amp is due to the fact that the closed-loop gain in the CFB configurations is changed by changing the input attenuation while maintaining a constant-loop gain. This has the implication that if the CFB amplifier should be designed to have a constant bandwidth this is only achieved at the cost of a decreased loop gain and bandwidth because the feedback resistor has to be fairly large. For designs optimized for bandwidth at a specific gain similar results can be expected from a conventional voltage mode op-amp and a CFB op-amp. However, a main attribute of the CFB op-amp is a high slew rate yielding a large signal bandwidth which is superior to most voltage mode op-amps. It should be pointed out, though, that the slew rate characteristics do not rely on the current feedback but rather on the internal architecture of the opamp. Recent evidence has been given [5], [6] that a similar slew rate performance can be achieved from voltage mode op-amps using an internal architecture resembling the architecture of the CFB op-amp.

\section{REFERENCES}

11] D. Nelson and S. Evans, "A new approach to op amp design," Comlinear Corporation Application Note 300-1, Mar. 1985.

[2] B. Wilson, “Analogue current mode circuits," Int. J. Electrical Engineering Education, vol. 26, pp. 206-233, 1989.

13] D. F. Bowers, "Applying 'current feedback' to voltage amplifiers," in C Toumazou, F. J. Lidgey, and D. G. Haigh, Eds., Analogue IC Design. The Current Mode Approach. London, UK: Peter Peregrinus, 1990, pp. 569-595.

[4] Analog Devices, AD844 Datasheet, 1990.

[5] E. Bruun, "High speed, current conveyor based voltage mode operational amplifier," Electron. Lett., vol. 28, pp. 742-744, Apr. 1992.

[6] E. Bruun, "A dual current feedback CMOS op amp," in Proc. Tenth NORCHIP Seminar, pp. A9-A11, Nov. 1992.

\section{Digital Clock Phase Shifter without a Phase Locked Loop}

\author{
John Cook
}

\begin{abstract}
A novel digitally controlled clock phase shifter is described that avoids the use of conventional phase locked loops with their attendant stability problems. The hardware is suitable for implementation as part of an integrated circuit. Two implementations are discussed, one of which of an integrated circuit. Two implementations are discussed, one of which
has low power consumption and is suitable for clocks of moderate speed and the second of which is suitable at higher frequencies. Simulation shows that the circuit is practically insensitive to component and timing tolerances. A prototype of the moderate speed version has been tested and shown results comparable with simulations.
\end{abstract}

\section{INTRODUCTION}

The problem that this phase shifter addresses is that of adjusting the phase of the clock in a digital system in small increments in such a way that there are no glitches produced and phase steps at all phases are approximately equal without the need to use a much higher intermediate frequency clock or to use a phase locked loop. In fact, the proposed system is entirely open loop.

One important application of such a phase shifter is in controlling the sampling phase of a digital transmission system receiver containing an echo canceller. There are two conventional solutions to this requirement, the use of an analog or a digital phase locked loop. In the analog version an independent oscillator has to be driven so that its phase tracks that of the incoming signal. As the oscillator control input affects its frequency the system being controlled is second order and the controller is notoriously difficult to design [1]-[3]. The design is often compromised by the need to accommodate the local oscillator center frequency tolerance (requiring a wide loop bandwidth) while the low output jitter needed for proper operation of the echo-canceller demands a narrow loop bandwidth. In the digital version usually only single clock cycle phase steps are possible and these are generally sufficiently large that their effect has to be compensated for in the echo canceller [4]-[6]. There are some known compromises in which the control of frequency and phase can be separated [7] but these still require the use of an independent oscillator.

The design problem is particularly acute at the master (clock controlling) end of a mesochronous duplex link because the effects of jitter are significant even if the jitter has a very low bandwidth, while the trend to ever higher transmission rates requires increasingly tight control over jitter. The difficulty over the center frequency accuracy of the independent oscillator is contemptible since the master clock is available and must by definition have exactly the required center frequency. The missing link is the lack of any convenient method to vary its phase as applied to one part of a circuit with respect to another. For the purposes of the echo canceller the phase of this clock must be variable in steps approximately three orders of magnitude smaller than the transmission system symbol period, and this over a range of, say, $+/-1$ symbol period. Although typically the local master clock may be much faster than the symbol rate in most cases this still means that single clock cycle phase steps are too large.

This paper describes an clock phase shifting technique that has an unlimited phase shift range and offers fine phase steps, all without

Manuscript received June 15, 1992; revised January 4, 1993. This paper was recommended by Associate Editor J. Choma.

J. Cook is with British Telecommunications Development and Procurement, Copper Access Systems Section, Martlesham, Ipswich, IPS 7RE, UK. IEEE Log Number 9208193 